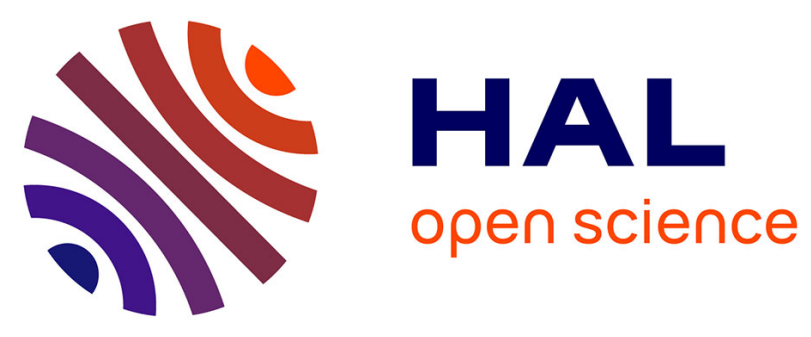

\title{
Metallic Nanodot Patterns with Unique Symmetries Templated from ABC Triblock Terpolymer Networks
}

Karim Aissou, Muhammad Mumtaz, Alberto Alvarez-Fernandez, Jean

Mercat, Ségolène Antoine, Gilles Pécastaings, Virginie Ponsinet, Cécile

Dobrzynski, Guillaume Fleury, Georges Hadziioannou

\section{To cite this version:}

Karim Aissou, Muhammad Mumtaz, Alberto Alvarez-Fernandez, Jean Mercat, Ségolène Antoine, et al.. Metallic Nanodot Patterns with Unique Symmetries Templated from ABC Triblock Terpolymer Networks. Macromolecular Rapid Communications, 2018, 39 (7), pp.1700754. 10.1002/marc.201700754 . hal-01688010

\section{HAL Id: hal-01688010 https://hal.science/hal-01688010}

Submitted on 2 Jul 2020

HAL is a multi-disciplinary open access archive for the deposit and dissemination of scientific research documents, whether they are published or not. The documents may come from teaching and research institutions in France or abroad, or from public or private research centers.
L'archive ouverte pluridisciplinaire HAL, est destinée au dépôt et à la diffusion de documents scientifiques de niveau recherche, publiés ou non, émanant des établissements d'enseignement et de recherche français ou étrangers, des laboratoires publics ou privés. 


\title{
Metallic Nanodot Patterns with Unique Symmetries Templated from
}

\author{
ABC Triblock Terpolymer Networks \\ Karim Aissou, ${ }^{1 *}$ Muhammad Mumtaz, ${ }^{1}$ Alberto Alvarez-Fernandez, ${ }^{1,2}$ Jean Mercat, ${ }^{1,3}$ \\ Ségolène Antoine, ${ }^{1}$ Gilles Pécastaings, ${ }^{1}$ Virginie Ponsinet, ${ }^{2}$ Cécile Dobrzynski, ${ }^{3}$ Guillaume \\ Fleury, ${ }^{1}$ and Georges Hadziioannou ${ }^{1}$ \\ ${ }^{1}$ Laboratoire de Chimie des Polymères Organiques (LCPO), CNRS - ENSCPB - Université de \\ Bordeaux, 16 Avenue Pey-Berland, F-33607 Pessac Cedex, France \\ ${ }^{2}$ Univ. Bordeaux, Centre de Recherche Paul Pascal (CRPP), CNRS UPR 8641, 115 avenue \\ Schweitzer, F-33600 Pessac, France \\ ${ }^{3}$ Bordeaux INP, IMB, UMR 5251/Inria Bordeaux Sud-Ouest, Team Cardamom, F-33405 \\ Talence cedex, France
}

E-mail: karim.aissou@enscbp.fr

ABSTRACT: Nanotemplates derived from the self-assembly of AB-type block copolymers provide an elegant route to achieve well-defined metallic dot arrays, even if, the variety of pattern symmetries is restricted due to the limited number of structures offered by microphase separated diblock copolymers. A strategy, that relies on the use of complex network structures accessible through the self-assembly of linear ABC-type terpolymers, is presented for the formation of metallic nanodots arrays with "outside-the-box" symmetries. Patterned templates formed by the cubic $Q^{214}$ and orthorhombic $O^{70}$ network structures were used as excellent platforms to build well-ordered gold nanodot arrays with unique $p 3 m 1$ and $p 2$ symmetries, respectively. A simple yet efficient blending strategy was used to tune the critical dimensions of the p3m1 pattern while laterally-ordered gold nanodot arrays were also demonstrated through a directed self-assembly approach. Such highly-ordered gold nanodots with tunable particle dimensions and array periods, enabling the control of their plasmonic responses, are attractive probes for biological imaging.

Production of perfectly-ordered metallic dot arrays at the nanometer scale on surfaces is always a hot topic in nanotechnology due to their unique and fascinating catalytic, electronic, optical and magnetic properties. For instance, dense magnetic nanodot (ND) arrays are highly desired for the next-generation of hard disk drives (HDDs) using a bit patterned media (BPM) concept to replace the conventional granular CoCrPt-oxide media, which becomes thermally unstable at areal densities of $\sim 1$ Tbit.in $^{-2} .^{[1]}$ On the other hand, noble metal NDs have the ability to resonantly scatter visible and near-infrared light upon the excitation of their surface plasmon 
oscillation. Hence, highly-ordered gold ND arrays have the potential to be used as nanobioplatforms for enhanced contrast cellular imaging. ${ }^{[2,3]}$ In addition, model patterned surfaces consisting of molecularly well-defined adhesive spots with dimension close to integrin diameters $(\sim 10 \mathrm{~nm})$ separated by non-adhesive regions are suitable for a wide range of biomedical applications based on the living cell adhesion. ${ }^{[4]}$ Gold dots functionalized with various thiol-bearing cell-adhesive peptides, due to the strong bonding between sulfur and Au, are excellent supports for the development of model peptide nanoscale arrays. Hovewer, the fabrication of metallic dots in high areal density with dimension on the order of $10 \mathrm{~nm}$ or less are chalenging to achieve with traditional lithography. ${ }^{[5]}$

As a result of microphase separation between the different blocks, self-assembled block copolymer thin films into well-ordered nanoscale morphologies ${ }^{[6-8]}$ such as spheres, cylinders, lamellae and complex networks are extremely versatile platforms to achieve well-defined metallic nanostructures. While AB-type block copolymer (BCP) systems offer an attractive route to produce ND arrays with a common $p 6 \mathrm{~mm}$ symmetry pattern due to packing frustration, linear ABC triblock terpolymers provide increased accessible structural diversity including core/shell versions of spheres or cylinders $(p 6 m m),{ }^{[9-13]}$ alternating spheres or cylinders with a tetragonal packing $(p 2 m m),{ }^{[14,15]}$ and three complex networks with patterns having $p 4 m m, p 3 m 1$ or $p 2$ symmetries. ${ }^{[16-21]}$

To create metallic ND patterns from self-assembled BCP thin films both ex-situ and insitu methodologies have been studied in the literature. ${ }^{[22-27]}$ The ex-situ route is an excellent approach to introduce multi-metallic nanoparticles (NPs) with controlled size and atomic arrangement (i.e. atomic structure with unique properties) within the BCP thin film acting as nanostructuring matrix. ${ }^{[22]}$ Using an ex-situ methodology, the precise positioning of metallic NPs on pupa-, pinecone-, bud-, and Janus-like BCP scaffolds was also achieved by tailoring the entropic and the enthalpic contributions within the emulsion droplet. ${ }^{[28-31]}$ In contrast, the in- 
situ route is straightforward to build mono-metallic NDs with a better control of the spacing arrangement since this route is based on a preferential incorporation of metallic precursors within the self-assembled BCP features. ${ }^{[26,32]}$ For instance, by using the in-situ methodology, well-ordered Au-Ag core-shell NP arrays, ${ }^{[33]}$ as well as complex structures consisting of bimetallic dots-on-wire nanostructures ${ }^{[34]}$ were demonstrated.

Although bulk network structures (i.e. $Q^{214}, Q^{230}$ and $\mathrm{O}^{70}$ structures of the space groups $I 4{ }_{1} 32, I a \overline{3} d$ and $F d d d$, respectively, where the $Q$ and $O$ annotations denote the cubic and orthorhombic phases) formed from linear ABC triblock terpolymers have demonstrated the potential to achieve 3D-continuous inorganic ${ }^{[35,36]}$ or metal ${ }^{[37]}$ structures, there has been no investigation of their thin film behavior nor their application in patterned template. Here, we report an in-situ BCP thin film approach to build well-ordered gold ND arrays on p3m1 and p2 symmetry patterns formed from two unique network nanostructures consisting of a cubic $\left(Q^{214}\right)$ alternating gyroid, $G^{\mathrm{A}}$, morphology (space group $14{ }_{1} 32$ ) and an orthorhombic $O^{70}$ phase (space group Fddd). In order to tune the dimension and the periodicity of gold nanodots ordered on a p3m1 pattern formed from the $G^{\mathrm{A}}$ structure, an efficient route based on the blend of different linear ABC triblock terpolymers is demonstrated. Finally, highly-oriented $p 3 m 1$ patterns were produced by templating the self-assembled $G^{\mathrm{A}}$ structures with topographical substrates.

\section{Results and Discussion}

Linear $\mathrm{ABC}$ triblock terpolymers consisting of poly(1,1-dimethyl silacyclobutane) (PDMSB, D), polystyrene (PS, S), and poly(2-vinyl pyridine) (P2VP, V) with molecular weights of 28 and $51 \mathrm{~kg} \cdot \mathrm{mol}^{-1}$ (noted DSV-28k and DSV-51k hereafter) were used in this work. These PDMSB- $b$-PS- $b$-P2VP terpolymers with narrow dispersities $(Đ<1.2)$ and specific compositions (D:S:V = 26:50:24 for DSV-28k and D:S:V = 32:41:28 for DSV-51k) were prepared by a sequential anionic polymerization of 1,1-dimethyl silacyclobutane (DMSB), 
styrene and 2-vinyl pyridine (2VP). To promote the self-assembly of blended and unblended DSV thin films, a post process solvent vapor annealing (SVA) with chloroform $\left(\mathrm{CHCl}_{3}\right)$ was performed.

Atomic force microscopy (AFM) topographic views presented in Figure 1a show a solvent-annealed (1h, $\left.\mathrm{CHCl}_{3}\right) 90 \mathrm{~nm}$ thick DSV-28k film, immersed into an aqueous $\mathrm{HAuCl}_{4}$ solution then subsequently treated by a $\mathrm{CF}_{4} / \mathrm{O}_{2}$ reactive ion etching (RIE) plasma, from which the characteristic (111) crystallographic plane of the $G^{\mathrm{A}}$ structure oriented parallel to the air surface can be observed. The $\mathrm{HAuCl}_{4}$-stained P2VP (bright) and etched PDMSB (dark) domains form a $p 3 m 1$ symmetry pattern with a period of $\sim 27 \mathrm{~nm}$. Scanning electron microscopy (SEM) images of the same sample revealed that the $\mathrm{CF}_{4} / \mathrm{O}_{2}$ RIE plasma simultaneously removes the PDMSB (dark) domains and partially reduces incorporated metallic precursors, resulting in a p3m1 symmetry pattern, in which the 3-fold Au NDs are surrounded by three larger holes. The associated grazing incidence small angle X-ray scattering (GISAXS) pattern mainly consists of intense Bragg rods resulting from the formation of ordered Au NDs on the DSV-28k film free surface (see Fig. S1a). In addition, the 2D-GISAXS pattern shows less intense peaks, consistent with 110 and 211 reflections of an alternating gyroid $\left(Q^{214}\right)$ structure (space group $I 4_{1} 32$ ) having a lattice constant of $\sim 35 \mathrm{~nm}$. The GISAXS pattern line-cut along $q_{\mathrm{y}}$ integrated between $q_{\mathrm{z}}$ values $0.037-0.041 \AA^{-1}$ revealed the position of a first-peak order at $q^{* *}=$ $0.013 \AA^{-1}$, corresponding to the 110 reflection of the $Q^{214}$ structure (see Fig. S1b). Moreover, the GISAXS profile indicates that Au NDs are highly-ordered into a hexagonal-close packed (HCP) array since a first Bragg rod maximum position at $q^{*}=0.025 \AA^{-1}$ (period of $\sim 28.9 \mathrm{~nm}$ in accordance with AFM and SEM images) and other Bragg peaks located at $q / q^{*}=1,3^{1 / 2}, 2,7^{1 / 2}$ and $9^{1 / 2}$ are clearly visible. The long-range order is fully supported by the simulated image obtained from a typical ( $4 \times 4 \mu \mathrm{m}$ ) AFM topographic image showing that the Au ND array contains free dislocations and dislocation lines delimiting large grains in a density of only 1.7 
\% (see Fig. S2). Such a long-range ordered $G^{\mathrm{A}}(111)$ pattern is an excellent template to achieve a gold dot array having large grains. In spite of the long-range order of the $Q^{214}$ phase, we can note that some P2VP domains are not perfectly formed within the structure which induces a certain level of distribution in size of the gold nanodots. This phenomenon suggests that the microphase-separation of DSV chains occurs in a two-steps mechanism as already observed for self-assembled ABC 3-miktoarm star terpolymer chains. ${ }^{[38]}$

In order to complete the reduction of metallic precursor within the P2VP domains without damaging the ND array, an additional oxygen RIE plasma $\left(60 \mathrm{~W}, 10 \mathrm{sccm} \mathrm{O}_{2}, \mathrm{XX}\right.$ mTorr, and 15 s) was also applied on the DSV-28k thin films as showed on the SEM image (see Fig. 2a) while the simulated results (see Fig. 2b) confirm the formation of a 2D array with large grains. To demonstrate an efficient reduction of $\mathrm{HAuCl}_{4}$ salt into metallic dots with $\mathrm{O}_{2} \mathrm{RIE}$ plasma, spectroscopic ellipsometry measurements were performed on stained and unstained DSV-28k thin films (see Fig. 2c) on the visible and near-IR spectral domain. Spectroscopic ellipsometry is a measurement technique providing access to the optical response of thin films with a high sensitivity. It measures the complex ellipsometric ratio $\rho=r_{\mathrm{p}} / r_{\mathrm{s}}$ where $r_{\mathrm{p}}$ and $r_{\mathrm{s}}$ are the amplitude reflection coefficients for light polarization parallel, $p$, and perpendicular, $s$, to the plane of incidence, and extracts the ellipsometric angles $\Psi$ and $\Delta$, with $\rho=\tan \Psi \mathrm{e}^{\mathrm{i} \Delta}$. The evolution of the measured $\Psi$ and $\Delta$ as a function of the photon energy revealed a clear S-shaped feature characteristic of a resonance in the region $\sim 2.36 \mathrm{eV}$, which is not present for the unstained DSV-28k thin film, and is attributed to the effect of the localized surface plasmon resonance of the Au dots templated from the P2VP domains. To control the grain orientation and improve their size within the $Q^{214}$ network nanostructure, topographical gratings in silica were used. These guiding patterns fabricated by interference lithography with a period of $1 \mu \mathrm{m}$ consisted of $700 \mathrm{~nm}$ width, $45 \mathrm{~nm}$ deep trenches separated by $300 \mathrm{~nm}$ width mesas. The AFM topographic image presented in Figure 2d shows a gold ND array with a single grain orientation 
(area of $2 \mu \mathrm{m}^{2}$ ) on topographical substrate as revealed by the six first-order spots on the 2DFFT. This highly-ordered gold array was produced from a templated $95 \mathrm{~nm}$ thick DSV-28k layer self-assembled into a $G^{\mathrm{A}}$ structure with the (111) plane oriented parallel to the air surface.

In order to control the periodicity of the $p 3 m 1$ symmetry pattern, the DSV-28k thin films were blended with different amounts of another DSV terpolymer with a higher molecular weight (D:S:V = 32:41:28, molecular weight = $51 \mathrm{~kg} \cdot \mathrm{mol}^{-1}$, noted DSV-51k). The SAXS pattern of the bulk DSV-51k sample shows a first-order peak, $q^{*}$, at $0.141 \mathrm{~nm}^{-1}$ and higher-order peaks located at $q / q^{*}=1,2,3,4,5,7$ and 8 , consistent with a lamellar phase having a period of $\sim 44.5$ nm (see Fig. S3). The evolution of the $G^{\mathrm{A}}(111)$ pattern period as a function of the DSV-51k mass fraction (noted $w_{\text {DSV-51k }}$ ) in the blend thin films is presented in Figure 3a. Period values of the blends, $p_{\text {blend, }}$ extracted from the GISAXS data provided in Figure S4 increase linearly with the DSV-51k mass fraction in the range of $0<w_{\text {DSV-51k }}<50$ wt. $\%$ ( $p_{\text {blend }} \sim 0.197 w_{\text {DSV }-51 k}$ + 29.1). For instance, Figure 3b shows SEM images of a solvent-annealed (1h, $\left.\mathrm{CHCl}_{3}\right) 70 \mathrm{~nm}$ thick DSV-28k film blended with 50 wt. \% of DSV-51k chains immersed into an aqueous $\mathrm{HAuCl}_{4}$ solution and then subsequently treated by a $\mathrm{CF}_{4} / \mathrm{O}_{2} \mathrm{RIE}$ plasma. The nanostructure has a p3m1 symmetry which consists of 3-fold Au NDs surrounded by three larger holes with a period of $\sim 38.7 \mathrm{~nm}$ in accordance with GISAXS measurements. Here, the blending strategy enables to finely adjust the period of the $G^{\mathrm{A}}(111)$ pattern as well as the P2VP domain size, which is of high interest for designing ordered metallic nanodot arrays on surfaces with tunable particle dimensions and array periods, likely to present for instance controlled plasmonic responses.

Interestingly, the DSV-28k morphology appeared to be very sensitive to the film thickness since an orthorhombic network with a $F d d d\left(O^{70}\right)$ space group was also formed during the solvent annealing process when the film thickness was increased. To illustrate this purpose, a $120 \mathrm{~nm}$ thick DSV-28k layer treated by a $\mathrm{CHCl}_{3}$ vapor during $1 \mathrm{~h}$ is shown in Figure 4. After 
etching the DSV-28k thin film with a fluorine-rich RIE plasma, the two morphologies could be clearly differentiated in the AFM topographic image shown in Figure 4a, where the dashed line delimits the upper and lower regions occupied by the $G^{\mathrm{A}}$ and $O^{70}$ structures, respectively. As depicted in the insets, [111] and [110] directions for the cubic and orthorhombic structures present patterns with $p 3 m 1$ and $p 2$ symmetries where the black and yellow dots correspond to the PDMSB and P2VP domains, respectively. Importantly, a positional match between the P2VP domains (yellow dots on the inset Fig. 4a) formed within the two patterns could be observed as indicated by the black meshes while the (red) primitive cell ( $a \sim 27 \mathrm{~nm}$ ) of the $\mathrm{G}^{\mathrm{A}}$ pattern along the [111] direction is exactly two times smaller than the one of the $O^{70}(110)$ projection. Gold dot arrays with $p 3 m 1$ and $p 2$ symmetries were also produced by treating the $\mathrm{HAuCl}_{4}$-stained P2VP domains with an $\mathrm{O}_{2}$ RIE plasma as showed on the SEM image presented in Figure 4b. The structure achieved from the $O^{70}$ structure appears as a line/space pattern where the lines are occupied by gold dots ordered into a $p 2$ array with dimensions very close to the pattern templated from the $G^{\mathrm{A}}$ structure. This “double-dot” pattern is consistent with the "U” shape formed by P2VP domains in the vicinity of the air surface within the $O^{70}$ structure. As observed in Figure 1b, the polydispersity of gold NDs suggests that the film ordered in a two-steps mechanism, and the microphase-separation of the P2VP domains occurring in a second step is not completed.

To provide more insight in the double-dot pattern, magnified AFM images showing the $\mathrm{O}^{70}$ structure were also studied. The AFM topographic image presented in Figure 5a shows the typical pattern observed from the [110] direction of the $\mathrm{O}^{70}$ structure after treating the $\mathrm{HAuCl}_{4}$ stained sample with a fluorine-rich plasma. The associated high resolution mapping corresponding to the adhesion properties confirmed that domains with an apparent lozenge-like shape on the topographical AFM image are indeed formed by two P2VP (black) domains, as revealed by the nanomechanical properties of the sample (see Fig. $\mathbf{5 b}$ ). As seen on the Figure 
5c, PS lines formed close to the DSV-28k film free surface have a period of $\sim 51 \mathrm{~nm}$ as extracted from the 2D-FFT (see also Fig. 5d), which indicates that the lattice constant $c$ of the orthorhombic unit cell is $\sim 102 \mathrm{~nm}$. The associated power spectral density (PSD) profile exhibits a first-order peak at $0.0193 \mathrm{~nm}^{-1}$ and higher-order peaks located at $q / q^{*}=1,1.71,1.86$ and 2 , consistent with a line/space pattern where the period of $\mathrm{HAuCl}_{4}$-stained P2VP domains along the PS lamellar direction ( $p \sim 29.9 \mathrm{~nm}$ ) is not exactly the same as the one in the opposite direction ( $p \sim 27.3 \mathrm{~nm}$ ) as represented in the Figure 5d. Importantly, the period along the PS lamellar direction corresponds to the lattice constant $a$ of the orthorhombic unit cell which indicates the $a: c$ ratio is very close to $1: 2 \sqrt{3}$ as predicted theoretically by Morse and Ranjan. ${ }^{[39]}$ Interestingly, a zigzag arrangement of P2VP domains is often observed on the pattern corresponding the [110] direction of the $O^{70}$ structure. To further the understanding of this phenomenon and the order-order transition (OOT) occurring between the $G^{\mathrm{A}}$ and $O^{70}$ structures, AFM images showing the two network structures will be discussed. The AFM topographic image presented in Figure 6a shows a region of the DSV-28k thin film where both the $G^{\mathrm{A}}$ and $\mathrm{O}^{70}$ structures coexist within the same grain, which are delimited by (black) dashed lines drawn on the image. The $O^{70}$ pattern which partially occupies the (green) grain presents a zigzagging structure where the U-shaped P2VP domains close to the air surface have a similar orientation. The zigzag arrangement of P2VP domains occurs during the early stage of the $O^{70}$ phase formation as revealed by the anisotropic small nuclei found on the AFM topographic image presented on Figure 6b. The schematic illustrates that the $O^{70}$ small nuclei consists of U-shaped P2VP domains which form a zigzagging structure within the $G^{\mathrm{A}}$ phase (see Fig. 6c). Epitaxial relations between the (111) and (110) planes of the $G^{\mathrm{A}}$ and $O^{70}$ structures are evident and the zigzag structure should appear on the early stage the $O^{70}$ phase formation as a kinetic pathway connecting the cubic and orthorhombic phases. 
The linear ABC triblock terpolymers used in this work are geometrically symmetric (i.e. equal volume of $\mathrm{A}$ and $\mathrm{C}$ blocks) with equivalent surface energies between the $\mathrm{A} / \mathrm{B}$ and $\mathrm{B} / \mathrm{C}$ interfaces (i.e. $\chi_{\mathrm{AB}} \approx \chi_{\mathrm{BC}}$ where $\chi$ represents the Flory-Huggins interaction parameter). Bulk self-assembly of such kind of $\mathrm{ABC}$ triblock terpolymer along the $\Phi_{\mathrm{A}}=\Phi_{\mathrm{C}}$ isopleth has been reported in the literature. ${ }^{[16,18,19]}$ For instance, Mogi et al. have demonstrated that the $G^{\mathrm{A}}$ and lamellar structures are formed in bulk from various polyisoprene- $b$-PS- $b$-P2VP (ISV) terpolymers having their styrenic mid-block volume fraction, $\Phi_{\mathrm{S}}$, in the ranges of $0.48<\Phi_{\mathrm{S}}<$ 0.66 and $0.08<\Phi_{\mathrm{S}}<0.42$, respectively. ${ }^{[16]}$ The morphologies of the DSV terpolymers studied in this work are in accordance with those occurring in bulk from ISV terpolymers since the thin film $G^{\mathrm{A}}$ (DSV-28k) and bulk lamellar (DSV-51k) structures have $\Phi_{\mathrm{S}}$ equal to 0.5 and 0.41 , respectively. These results are also in good agreement with self-consistent field theory (SCFT)

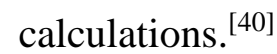

Importantly, the thin film $G^{\mathrm{A}}$ structure becomes unstable when the film thickness is increased since an epitaxial growth of an orthorhombic $O^{70}$ network with a space group Fddd on $G^{\mathrm{A}}(111)$ pattern occurs within thicker polymeric layers during the SVA process. This orderdisorder transition (OOT) occurs only when the DSV thin film is exposed to a continuous stream with a high $\mathrm{CHCl}_{3}$ vapor pressure in the chamber, swelling the polymer layers without dewetting. Indeed, the $Q^{214}$ to $O^{70}$ OOT was observed when the continuous flow system used to control the $\mathrm{CHCl}_{3}$ vapor pressure in the chamber by dilution with a separate $\mathrm{N}_{2}$ stream was set so that the solvent vapor consisted of $32 \mathrm{sccm} \mathrm{CHCl}_{3}$ vapor and $8 \mathrm{sccm} \mathrm{N}_{2}$ (total $40 \mathrm{sccm}$ ). In contrast, the OOT was not observed when the solvent vapor consisted of $16 \mathrm{sccm} \mathrm{CHCl}_{3}$ vapor and $4 \mathrm{sccm} \mathrm{N}_{2}$ (total $20 \mathrm{sccm}$ ), even for long exposition times (ranging from 1 to $17 \mathrm{~h}$ ). As melt-phase OOTs from $Q^{214}$ to $O^{70}$ upon heating have been reported for several PI- $b$-PS- $b$ poly(ethylene oxide) (ISO) terpolymers $\left(\chi_{\mathrm{IS}} \approx \chi_{\mathrm{SO}}\right)$ with compositions close to the $\Phi_{\mathrm{I}}=\Phi_{\mathrm{O}}$ isopleth, we expect that the OOT occurring under a high $\mathrm{CHCl}_{3}$ vapor pressure is mainly due 
to a decrease of the effective Flory-Huggins interaction parameters, $\chi_{\text {eff, }}$ in the presence of solvent. ${ }^{[18,19]}$ This is also in accordance with theoretical results obtained by Tyler and Morse, who showed that similarities between the $O^{70}$ and $Q^{214}$ phases as 3-fold networks, make the $O^{70}$ network a competitor to the $Q^{214}$ network, which is apparently preferred over the $Q^{214}$ phase in the limit of weak segregation (i.e. when $\chi$ is decreased). ${ }^{[41]}$

\section{Conclusion}

In-situ fabrication of metallic nanodot arrays on surfaces from BCP templates have been extensively studied in the literature, however no investigation have demonstrated the use of network-forming BCP thin films as patterned templates. Network structures formed from ABC terpolymer thin films offer access to "three-colored” patterns which have been used as excellent platforms to create highly-ordered metallic ND arrays with unique symmetries. For instance, gold ND arrays with $p 3 m 1$ and $p 2$ symmetries have been produced from cubic and orthorhombic network structures. Particularly, line/space gratings with an ordered metallic double-dot pattern have been fabricated from the $\mathrm{O}^{70}$ network. The precise control of the ND positions within a line/space pattern, which is challenging with state-of-the-art lithography techniques, could find application in multi-bit high-density memory devices.

\section{Methods}

Self-assembly of DSV thin films: Untemplated and templated DSV thin films were spincoated (2-5 krpm) using a 2 wt. \% copolymer solution in a mixture of tetrahydrofuran and propylene glycol monomethyl ether acetate (THF/PGMEA: 2/1). The self-assembly of DSV thin films was promoted by exposing samples for different times (ranging from $1 \mathrm{~h}$ to $17 \mathrm{~h}$ ) to a continuous stream of $\mathrm{CHCl}_{3}$ vapor produced by bubbling nitrogen gas through the liquid solvent as described previously. ${ }^{[42]}$ This continuous flow system was used to control the $\mathrm{CHCl}_{3}$ vapor pressure in the chamber by dilution with a separate $\mathrm{N}_{2}$ stream so that a solvent vapor consisted 
of $32 \mathrm{sccm} \mathrm{CHCl}_{3}$ vapor and 8 sccm $\mathrm{N}_{2}$ swelled the films without dewetting. Both templated and untemplated film thicknesses were determined by AFM from scratched polymer areas.

Topographical grating fabrication: The periodic topographical gratings were fabricated using a Lloyd's mirror interferometer with a $325 \mathrm{~nm}$ He-Cd laser beam. To transfer the interference lithography pattern into the $\mathrm{Si} / 45 \mathrm{~nm} \mathrm{SiO}$ layer, a trilayer resist stack methodology was used. This trilayer was obtained by inserting a hard mask layer consisting of a $\mathrm{SiO}_{2}$ thick film between a chemically-amplified negative resist resin and an anti-reflection coating (ARC) layer. After development of the resist, the pattern was transferred into the hard mask then into the ARC and finally into the $\mathrm{SiO}_{2}$ by a series of RIE steps to form a grating.

SEM and AFM Characterizations: Scanning electron microscopy (SEM, JEOL 7800) was used in Gentle Beam mode (GBSH) at an accelerating voltage of $1 \mathrm{kV}$ to take DSV thin film images. Atomic force microscopy (AFM Dimension FastScan, Bruker) was used in tapping mode to characterize the surface morphology of DSV thin films. Silicon cantilevers (FastscanA) with a typical tip radius of $\sim 5 \mathrm{~nm}$ were used. The resonance frequency of the cantilevers was about $1.25 \mathrm{kHz}$. Prior to SEM and AFM measurements, DSV thin films were immersed into an aqueous $\mathrm{HAuCl}_{4}$ solution (1 wt. \%, 2h) then subsequently etched with a fluorine-rich plasma treatment in PE-100 chamber (RIE, Plasma Etch) to remove preferentially the PDMSB phase (plasma conditions: $40 \mathrm{~W}, 17$ sccm $\mathrm{CF}_{4}$ and 3 sccm O 2,180 mTorr, and 45 s). An additional, $\mathrm{O}_{2}$ RIE plasma was also applied on several samples (plasma conditions: $60 \mathrm{~W}, 10$ sccm $\mathrm{O}_{2}, \mathrm{XX}$ mTorr, and $15 \mathrm{~s}$ ) in order to complete the $\mathrm{Au}^{3+}$ to $\mathrm{Au}^{0}$ reduction.

Automated structural analysis: AFM and SEM images of the nanodots analysis were automated using the Crysa software. Crysa is a software that automatizes 2D CRYstal Structural Analysis from an image. It was used to recover the dot positions in the images and produce their associated Delaunay triangulations. That enabled defect recognition and grain orientation 
measurement as well as graphical output production. Curves and global indicators such as pair correlation, grain sizes and mean dot spacing were used for array ordering characterization.

Spectroscopic Ellipsometry: Spectroscopic Ellipsometry was performed in reflection with a UVISEL phase-modulated ellipsometer (from Horiba Scientific) over the spectral range $[0.6-4.5 \mathrm{eV}]$ and with an angle of incidence of $60^{\circ}$. We extracted the two ellipsometric angles $\Psi$ and $\Delta$ from the measure of $I_{\mathrm{s}}=\sin 2 \Psi \sin \Delta$ and $I_{\mathrm{c}}=\sin 2 \Psi \cos \Delta$, in the UVISEL II $(\mathrm{P}=45$; $\mathrm{M}=0$ ) configuration.

GISAXS Measurements: GISAXS experiments were performed on the Dutch-Belgian Beamline (DUBBLE) at the European Synchrotron Radiation Facility (ESRF) station BM26B in Grenoble $(12 \mathrm{keV}) \cdot{ }^{[4]}$ The incidence angle was set at $0.14^{\circ}$, which is between the critical angle of the DSV film and the silicon substrate, to illuminate (by the beam) the samples with a typical size of $150 \mathrm{~mm}^{2}$. 2D scattering patterns were collected with a PILATUS $1 \mathrm{M}$ detector and the sample-to-detector distance was set to $3700 \mathrm{~mm}$. Unblended and blended DSV samples for GISAXS measurements were spin-coated onto Si substrates from a 2 wt. \% copolymer solution in a mixture of THF/PGMEA (2/1) then exposed to a $\mathrm{CHCl}_{3}$ vapor to promote the DSV thin film self-assembly.

\section{Acknowledgements}

The authors acknowledge support from Arkema and the Nouvelle Région Aquitaine and from the LabEx AMADEus (ANR-10-LABX-42) in the framework of IdEx Bordeaux (ANR-10IDEX-03-02), France. The ESRF and NWO are acknowledged for allocating beamtime at the Dutch-Belgian beamline (DUBBLE) for the GISAXS experiments. This work was performed within the framework of the Equipex ELORPrintTec ANR-10-EQPX-28-01 with the help of the LCPO-Arkema INDUSTRIAL CHAIR “HOMERIC” ANR-13-CHIN-0002-01. 


\section{REFERENCES}

[1] S. N. Piramanayagam, K. Srinivasan, J. Magn. Magn. Mater. 2009, 321, 485.

[2] K. Sengupta, E. Moyen, M. Macé, A.-M. Benoliel, A. Pierres, F. Thibaudau, L. Masson, L. Limozin, P. Bongrand, M. Hanbücken, Small 2009, 5, 449.

[3] M. Jung, W. A. El-Said, J.-W. Choi, Nanotechnology 2011, 22, 235304.

[4] M. Arnold, E. A. Cavalcanti-Adam, R. Glass, J. Blümmel, W. Eck, M. Kantlehner, H. Kessler, J. P. Spatz, ChemPhysChem 2004, 5, 383.

[5] Y. Luo, Y. Du, V. Misra, Nanotechnology 2008, 19, 265301.

[6] F. S. Bates, G. H. Fredrickson, Annu. Rev. Phys. Chem. 1990, 41, 525.

[7] M. W. Matsen, M. Schick, Phys. Rev. Lett. 1994, 72, 2660.

[8] M. W. Matsen, Macromolecules 2012, 45, 2161.

[9] S. Guo, J. Rzayev, T. S. Bailey, A. S. Zalusky, R. Olayo-Valles, M. A. Hillmyer, Chem. Mater. 2006, 18, 1719.

[10] V. P. Chuang, C. A. Ross, P. Bilalis, N. Hadjichristidis, ACS Nano 2008, 2, 2007.

[11] V. P. Chuang, C. A. Ross, J. Gwyther, I. Manners, Adv. Mater. 2009, 21, 3789.

[12] M. D. Rodwogin, A. Baruth, E. A. Jackson, C. Leighton, M. A. Hillmyer, ACS Appl. Mater. Interfaces 2012, 4, 3550.

[13] K. Aissou, M. Mumtaz, P. Marcasuzaa, C. Brochon, E. Cloutet, G. Fleury, G. Hadziioannou, Small 2017, 13, 1603184.

[14] V. P. Chuang, J. Gwyther, R. A. Mickiewicz, I. Manners, C. A. Ross, Nano Lett. 2009, 9, 4364.

[15] J. G. Son, J. Gwyther, J.-B. Chang, K. K. Berggren, I. Manners, C. A. Ross, Nano Lett. 
2011, 11, 2849.

[16] Y. Mogi, M. Nomura, H. Kotsuji, K. Ohnishi, Y. Matsushita, I. Noda, Macromolecules 1994, 27, 6755.

[17] T. S. Bailey, C. M. Hardy, T. H. Epps, F. S. Bates, Macromolecules 2002, 35, 7007.

[18] T. H. Epps, T. S. Bailey, R. Waletzko, F. S. Bates, Macromolecules 2003, 36, 2873.

[19] T. H. Epps, E. W. Cochran, C. M. Hardy, T. S. Bailey, R. S. Waletzko, F. S. Bates, I. Thomas H. Epps, † Eric W. Cochran, Cordell M. Hardy, Travis S. Bailey, and Ryan S. Waletzko, F. S. Bates*, Macromolecules 2004, 37, 7085.

[20] J. Chatterjee, S. Jain, F. S. Bates, Macromolecules 2007, 40, 2882.

[21] T. A. Shefelbine, M. E. Vigild, M. W. Matsen, D. A. Hajduk, M. A. Hillmyer, E. L. Cussler, F. S. Bates, J. Am. Chem. Soc. 1999, 121, 8457.

[22] K. Aissou, T. Alnasser, G. Pecastaings, G. Goglio, O. Toulemonde, S. Mornet, G. Fleury, G. Hadziioannou, J. Mater. Chem. C 2013, 1, 1317.

[23] M. R. Bockstaller, R. A. Mickiewicz, E. L. Thomas, Adv. Mater. 2005, 17, 1331.

[24] Y. Lin, A. Böker, J. He, K. Sill, H. Xiang, C. Abetz, X. Li, J. Wang, T. Emrick, S. Long, Q. Wang, A. Balazs, T. P. Russell, Nature 2005, 434, 55.

[25] M. Aizawa, J. M. Buriak, J. Am. Chem. Soc. 2005, 127, 8932.

[26] H. Cho, H. Park, T. P. Russell, S. Park, J. Mater. Chem. 2010, 20, 5047.

[27] D. O. Shin, D. H. Lee, H.-S. Moon, S.-J. Jeong, J. Y. Kim, J. H. Mun, H. Cho, S. Park, S. O. Kim, Adv. Funct. Mater. 2011, 21, 250.

[28] N. Yan, Y. Zhang, Y. He, Y. Zhu, W. Jiang, Macromolecules 2017, 50, 6771.

[29] Y. Zhang, Y. He, N. Yan, Y. Zhu, Y. Hu, J. Phys. Chem. B 2017, 121, 8417.

[30] N. Yan, H. Liu, Y. Zhu, W. Jiang, Z. Dong, Macromolecules 2015, 48, 5980. 
[31] S. G. Jang, D. J. Audus, D. Klinger, D. V. Krogstad, B. J. Kim, A. Cameron, S.-W. Kim, K. T. Delaney, S.-M. Hur, K. L. Killops, G. H. Fredrickson, E. J. Kramer, C. J. Hawker, J. Am. Chem. Soc. 2013, 135, 6649.

[32] M. S. Onses, L. Wan, X. Liu, N. B. Kiremitler, H. Yılmaz, P. F. Nealey, ACS Macro Lett. 2015, 4, 1356.

[33] S. K. Cha, J. H. Mun, T. Chang, S. Y. Kim, J. Y. Kim, H. M. Jin, J. Y. Lee, J. Shin, K. H. Kim, S. O. Kim, ACS Nano 2015, 9, 5536.

[34] Z. Liu, T. Chang, H. Huang, T. He, ACS Appl. Mater. Interfaces 2015, 7, 25938.

[35] M. Stefik, S. Mahajan, H. Sai, T. H. Epps, F. S. Bates, S. M. Gruner, F. J. DiSalvo, U. Wiesner, Chem. Mater. 2009, 21, 5466.

[36] E. J. W. Crossland, M. Kamperman, M. Nedelcu, C. Ducati, U. Wiesner, D.-M. Smilgies, G. E. S. Toombes, M. A. Hillmyer, S. Ludwigs, U. Steiner, H. J. Snaith, Nano Lett. 2009, 9, 2807.

[37] S. Salvatore, A. Demetriadou, S. Vignolini, S. S. Oh, S. Wuestner, N. A. Yufa, M. Stefik, U. Wiesner, J. J. Baumberg, O. Hess, U. Steiner, Adv. Mater. 2013, 25, 2713.

[38] K. Aissou, A. Nunns, I. Manners, C. A. Ross, Small 2013, 9, 4077.

[39] A. Ranjan, D. C. Morse, Phys. Rev. E 2006, 74, 11803.

[40] M. W. Matsen, J. Chem. Phys. 1998, 108, 785.

[41] C. A. Tyler, J. Qin, F. S. Bates, D. C. Morse, Macromolecules 2007, 40, 4654.

[42] K. W. Gotrik, A. F. Hannon, J. G. Son, B. Keller, A. Alexander-Katz, C. A. Ross, ACS Nano 2012, 6, 8052.

[43] G. Portale, D. Hermida-Merino, W. Bras, Eur. Polym. J. 2016, 81, 415. 
Figure 1: (a) AFM topographic and (b) SEM views of a solvent-annealed (1h, $\mathrm{CHCl}_{3}$ ) DSV28k thin film $(t \sim 90 \mathrm{~nm})$ immersed into an aqueous $\mathrm{HAuCl}_{4}$ solution then subsequently treated by a $\mathrm{CF}_{4} / \mathrm{O}_{2}$ RIE plasma where partially reduced $\mathrm{HAuCl}_{4}$-stained P2VP (bright) and etched PDMSB (dark) domains are arranged in a $p 3 m 1$ symmetry pattern of $\sim 27 \mathrm{~nm}$ period. Scale bars: $200 \mathrm{~nm}$.

Figure 2: (a) SEM image and (b) its associated simulated results of a solvent-annealed (1h, $\left.\mathrm{CHCl}_{3}\right)$ DSV-28k thin film ( $t \sim 90 \mathrm{~nm}$ ) immersed into an aqueous $\mathrm{HAuCl}_{4}$ solution then subsequently treated by a two-step sequential $\mathrm{CF}_{4} / \mathrm{O}_{2}$ and $\mathrm{O}_{2} \mathrm{RIE}$ plasma process. In the Delaunay triangulation, small dots are 6-fold coordinated P2VP domains while black and white big dots, indicating defect sites, are five-, and seven-fold coordinated P2VP domains, respectively. The colormap indicates the grain orientations $\left(0-60^{\circ}\right)$. (c) Spectroscopic ellipsometry results obtained from $\mathrm{HAuCl}_{4}$-stained and unstained DSV-28k thin films $(t \sim 90$ $\mathrm{nm}$ ) treated by a two-step sequential $\mathrm{CF}_{4} / \mathrm{O}_{2}$ and $\mathrm{O}_{2} \mathrm{RIE}$ plasma process. (d) Au nanodot array produced from a templated $95 \mathrm{~nm}$ thick DSV-28k film immersed into an aqueous $\mathrm{HAuCl}_{4}$ solution then subsequently treated by a two-step sequential $\mathrm{CF}_{4} / \mathrm{O}_{2}$ and $\mathrm{O}_{2}$ RIE plasma process. The polymeric film thicknesses were $\sim 55 \mathrm{~nm}$ on mesas and $\sim 90 \mathrm{~nm}$ in grooves. Scale bars: 200 $\mathrm{nm}$.

Figure 3: (a) Evolution of the $G^{\mathrm{A}}$ (111) pattern period as a function of $w_{\mathrm{DSV}}$-51k in the blend thin films. (b) SEM image of a solvent-annealed (1h, $\left.\mathrm{CHCl}_{3}\right) \mathrm{DSV}-28 \mathrm{k}$ thin film $(t \sim 70 \mathrm{~nm})$ blended with 50 wt. \% of DSV-51k after immersion into an aqueous $\mathrm{HAuCl}_{4}$ solution and subsequent treatment by a $\mathrm{CF}_{4} / \mathrm{O}_{2} \mathrm{RIE}$ plasma. The blend thin film presents a $p 3 m 1$ symmetry pattern of $\sim 38.7 \mathrm{~nm}$ period. Scale bars: $200 \mathrm{~nm}$.

Figure 4: (a) (left) low and (right) high magnification AFM topographic views of a solventannealed (1h, $\left.\mathrm{CHCl}_{3}\right) \mathrm{DSV}-28 \mathrm{k}$ thin film $(t \sim 120 \mathrm{~nm})$ treated by a $\mathrm{CF}_{4} / \mathrm{O}_{2} \mathrm{RIE}$ plasma. The dashed line delimits the upper and lower regions occupied by the $G^{\mathrm{A}}$ and $O^{70}$ structures, respectively. On schematics, the black and yellow dots indicate the PDMSB and P2VP domain positions on the $p 3 m 1$ and $p 2$ symmetry patterns while the different primitive cells are displayed in red. (b) (left) low and (right) high magnification SEM pictures of a solvent-annealed (1h, $\left.\mathrm{CHCl}_{3}\right)$ DSV-28k thin film $(t \sim 120 \mathrm{~nm})$ immersed into an aqueous $\mathrm{HAuCl}_{4}$ solution then subsequently treated by a two-step sequential $\mathrm{CF}_{4} / \mathrm{O}_{2}$ and $\mathrm{O}_{2} \mathrm{RIE}$ plasma process. The dashed line delimits the upper and lower regions occupied by the $O^{70}$ and $G^{\mathrm{A}}$ structures, respectively. Scale bars: $200 \mathrm{~nm}$. 
Figure 5: (a) AFM topographic view and (b) its associated high resolution mapping corresponding to the adhesion properties of a solvent-annealed (1h, $\mathrm{CHCl}_{3}$ ) DSV-28k thin film $(t \sim 120 \mathrm{~nm})$ immersed into an aqueous $\mathrm{HAuCl}_{4}$ solution then subsequently treated by a $\mathrm{CF}_{4} / \mathrm{O}_{2}$ RIE plasma. (c) 2D-FFT and its associated PSD profile extracted from (a). (d) Schematic showing the partially reduced $\mathrm{HAuCl}_{4}$-stained P2VP domain positions (yellow dots) and giving the characteristic dimensions of the pattern observed from the [110] direction of the $O^{70}$ structure. Scale bars: $200 \mathrm{~nm}$.

Figure 6: (a) low and (b) magnified AFM topographic views of a solvent-annealed (1h, $\mathrm{CHCl}_{3}$ ) DSV-28k thin film ( $t \sim 120 \mathrm{~nm})$ immersed into an aqueous $\mathrm{HAuCl}_{4}$ solution then subsequently treated by a $\mathrm{CF}_{4} / \mathrm{O}_{2} \mathrm{RIE}$ plasma. The colormap indicates the grain orientations $\left(0-60^{\circ}\right)$. (c) Schematic showing the P2VP domain positions observed in (b): isolated and connected dots correspond to P2VP domains within the $G^{\mathrm{A}}$ and $O^{70}$ structures. Scale bars: $200 \mathrm{~nm}$.

\section{Use for table of content only}

